\title{
CCP4 Web Services and Cloud Computing Developments
}

\author{
Ville Uski, Eugene Krissinel, and Charles Ballard \\ Research Complex at Harwell, Didcot, OX14 4 AS Oxfordshire, UK \\ ville.uski@stfc.ac.uk
}

The Collaborative Computational Project Number 4 in Protein Crystallography (CCP4) exists to maintain, develop and provide world-class software that allows researchers to determine macromolecular structures by X-ray crystallography and other biophysical techniques. Already for 38 years, the CCP4 Software has been assembled and distributed as an integrated Suite of programs, installable on either users' personal PCs or centralized facilities. The Suite is traditionally operated via CCP4i(2) Graphical User Interface and is available for all major Linux, Mac OSX and Windows platforms.

Modern trends in computing suggest a fast-growing interest to mobile platforms and cloud solutions for data storage and operations in practically all areas. These trends are observed in both hardware (such as the appearance of Chromebook-like and tablet devices) and operating systems, which now routinely include a number of cloud services from their vendors, changing the pattern of ordinary computing. In context of crystallographic computing, cloud solutions become increasingly appealing also in view of recent advances in automated structure solution methods, which are demanding for both computing power and various databases, making them less convenient for offline setups. Yet another reason for mobile trend to persist is that the cloud model of operations simplifies software and data management for both software provider and end users. Although CCP4 invested a considerable effort into development and maintenance of its dynamic update system, keeping both software and data resources in sync proves to be a burden for many users with limited computer or Internet resources.

CCP4 steps into the area of mobile computing and cloud services in 3 different ways. Firstly, it provides a set of free web services for automated structure solution. In many cases, an upload of reflection data and sequence is all what a user needs to do in order to solve their structure. Secondly, a system of cloud-based virtual machines with per-user persistent storage, access to collected data at DLS synchrotron and pre-installed CCP4 software is under development to serve computing needs of MX community in the UK. Thirdly, RESTful API is being developed, which will provide access to remote CCP4 computing on script level. Further plans include the development of native HTML5 interface for CCP4 Software, which would be useable on all mobile devices. We will discuss these developments, achieved results and future directions in middle-term perspective. 